\title{
Identification of Key Genes and Competitive Endogenous RNA Network Construction in Osteonecrosis of the Femoral Head by Integrated Bioinformatics Analysis
}

\section{Yang Dinglong}

The second hospital of Shanxi medical university

Chen Shuai

the second hospital of Shanxi medical university

Chen Yujing

the second hospital of Shanxi medical university

\section{Wang Beiyang}

the second hospital of Shanxi medical university

\section{Zhang Guohao}

the second hospital of Shanxi medical university

Zhang Zhiqiang ( $\sim$ zzq19834514218@163.com)

The second hospital of Shanxi medical university https://orcid.org/0000-0002-9288-5832

\section{Research article}

Keywords: Osteonecrosis of the femoral head (ONFH), ceRNA, PLEK, TLR2, TREM1

Posted Date: November 15th, 2021

DOI: https://doi.org/10.21203/rs.3.rs-1050652/v1

License: (c) (i) This work is licensed under a Creative Commons Attribution 4.0 International License.

Read Full License 


\section{Abstract}

Background: Despite cumulative evidence shows osteonecrosis of the femoral head (ONFH) could result in the progressive collapse of the femoral head. The pathogenesis of ONFH remains unclear. Early ONFH is difficult to diagnose due to the lack of effective biomarkers.

Method: In Gene Expression Omnibus (GEO) database, we searched the Microarray datasets for serum (GSE123568) in ONFH and normal controls to identify differentially expressed genes (DEGs) by R software. The enrichment analyses were performed to enrich pathways of DEGs. Protein-protein interaction (PPI), miRNA-mRNA co-expression, ceRNA networks were constructed using Cytoscape to identity top 15 hub genes, target miRNAs of hub genes and potential regulatory pathways. Furthermore, hub genes validated in GSE74089 with high diagnostic value for ONFH were selected as key genes. The Human Protein Atlas (HPA) and Bgee Database were used to find out the subcellular and tissue distribution of key genes.

Results: A total of 568 DEGs were identified between 30 ONFH samples and 10 normal controls. Kyoto Encyclopedia of Genes and Genomes (KEGG) and Gene Ontology (GO) enrichment analysis showed that DEGs are mostly enriched in innate immune responses, thrombosis and signal transduction. Fifteen hub genes were identified by PPI network using Cytoscape. The 15 hub genes were almost all positively

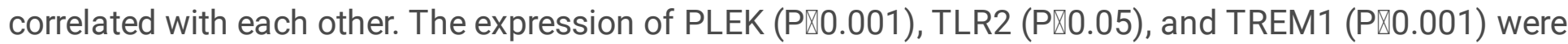
validated in dataset GSE74089 and they had high diagnostic value (AUC『0.8) for ONFH. MALAT1-miR146b-5p-TLR2, MALAT1-miR-664b-3p-PLEK, NORAD-miR-106b-5p-TLR2, and MSM01-miR-106b-5p-TLR2 might be potential RNA regulatory pathways in the disease progression of ONFH. PLEK mainly expressed in nucleus, TREM1 in dictyosome, and TLR2 in nucleoplasm and mitochondria.

Conclusions: In this study, we found that PLEK, TLR2, and TREM1 might be potential biomarkers in diagnostic and play a vital role in the progression of ONFH.

\section{Introduction}

Osteonecrosis of the femoral head (ONFH) is a potentially debilitating disease that results in the progressive collapse of the femoral head and subsequent degenerative arthritis (1). ONFH is a relatively common disorder that most frequently occurs in men aged 30-50 years with several major risk factors, including trauma, long-term or high-dose use of glucocorticoids, and long-term heavily intake of alcohol. In the United States, more than 20,000 new cases of femoral head necrosis are diagnosed each year (2). From 1992 through 2008 in the United States, the total number of surgeries for ONFH increased from 3570 to 6400 per year, nearly doubling over a 16-year time period (3). Among 8.12 million patients have been diagnosed with ONFH as of 2017 in China, and the average annual number of new cases was 14,103 in Korea (4). However, the pathogenesis of ONFH is still unclear (5).

As a subtype of non-traumatic ONFH, steroid-induced ONFH (SONFH) is related to the elevation of pressure in bone because of increasing lipogenesis and hyperplasia of adipocytes in bone marrow. 
Increased pressure could decelerate the blood flow in femoral head eventually causing avascular osteonecrosis (6). Bone marrow edema, decreased hematopoietic cells, adipocyte hypertrophy and a marked increase in empty bone traps in the subchondral region of the femoral head suggests that blood hyperviscosity may play an important role in the pathogenesis of hormonal ischemic necrosis of the femoral head (7). Because of nonspecific symptom in the early period, patients with SONFH usually miss the best time to receive nonsurgical therapy, early diagnosis and treatment of SONFH are crucial (8). Many ONFH patients with nonspecific symptoms often lead to late detection. Late stage of ONFH will seriously affect the life quality of patients for its pain or other reasons. However, there is no effective drug to treat ONFH at an early stage. Until now, effective biomarkers for diagnosis of ONFH remains lacking. The etiology of traumatic ONFH has been specific, the molecular mechanisms and pathogenesis underlying non-traumatic ONFH remains unclear, which is the basics for the molecular therapy of ONFH.

Currently, microarray and transcriptomic analyses have been widely used in various diseases, including a variety of tumors and ONFH (9-11), to identify new biomarkers to improve treatment and diagnosis. In addition, competitive endogenous RNA (ceRNA) networks can elucidate a new mechanism for promoting the development of the disease in a transcriptional regulatory network (12). However, transcriptional regulatory network for $\mathrm{ONFH}$ remains lacking. Through the combination of microarray and bioinformatics analyses, it is possible to explore potential vital genes and pathway networks that are closely related to the development of diseases.

In this study, based on Gene Expression Omnibus (GEO) database, we first identified differentially expressed genes (DEGs) by screening criteria. Then Gene Ontology (GO) and Kyoto Encyclopedia of Genes and Genomes (KEGG) enrichment analyses were performed. A protein-protein interaction (PPI) network was constructed to identify clustering modules and hub genes associated with ONFH. Further, target miRNAs of selected hub genes were predicted, and co-expression networks were constructed. Subsequently, we screened the selected hub genes using another GEO dataset and receiver operating characteristic (ROC) curve. Finally, we constructed ceRNA networks based on the predictions of long noncoding RNAs (IncRNAs) and circular RNAs (circRNAs) (Fig. 1).

\section{Methods}

\section{GEO data acquisiton}

We used GEO database to obtain microarray data for ONFH patients and normal controls. Screening criteria: (1) Homo sapiens Expression Profling by array; (2) phripheral secrum, hip cartilage or femoral head tissues of ONFH patients and normal controls; (3) more than five samples were included in each dataset; (4) complete information about samples in each dataset. Finally, the GPL15207 dataset GSE123568, which included phripheral securm samples in 30 ONFH patients and 10 normal controls, was selected as test sets. The GPL13497 dataset GSE74089 obtaining 4 ONFH and 4 non-ONFH hip cartilage samples was selected as validation set. 


\section{Data normalization and identification of DEGs}

The original files were downloaded from the GEO database, and pre-processed by using R software (version 3.6.3) GEO query package (13), and the probes that targeted multiple molecules were screened. The box diagrams were used to show the degree of inter-sample normalization. The limma package was used to analyse the differentially expressed genes. The screening criteria were $\|$ log2 (fold change) $\mid>1$ and adjusted $p$ value ( $Q$ value) $<0.05$. To better visualize these $D E G s$, we used $R$ software Complex Heatmap package to make heatmaps and ggplot2 package to make volcano plots.

\section{Enrichment analysis}

Gene Set Enrichment Analysis (GSEA) was used to analyse the distribution trends of the DEGs in a predefined set to determine their contribution to phenotypes. R software (version 3.6.3) clusterProfile package (14) was used for GSEA, org.Hs.eg.db package for ID translation and GOplot package for Zscore calculation (15). And the histogram, bubble diagram, ring diagram and chordal graph were created to visualize these enrichment results. The enriched pathways or functions with false discovery rate (FDR)

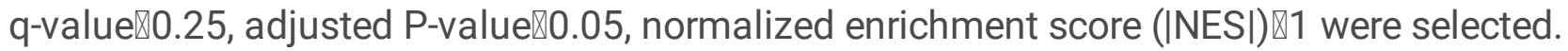

\section{Construction of the PPI network}

The online tool STRING (https://string-db.org/) with a filter condition (combined score『0.4) was used to constructed PPI network based on all DEGs. Next, the PPI network was generated in Cytoscape software (v3.8.2) using the downloaded interaction information. Minimal Common Oncology Data Elements (MCODE) was used to obtain cluster scores and identify important gene clusters. CytoHubba was used to recognize the hub genes which were located toward the network center (16). We used namely Degree, five algorithms, Maximum Neighborhood Component (MNC), Maximal Clique Centrality (MCC), Clustering Coefcient, and Density of Maximum Neighborhood Component (DMNC) to calculate the top 15 hub genes.

\section{Construction of the miRNA-mRNA and protein-chemical interaction networks}

We used NetworkAnalyst database (https://www.networkanalyst.ca/) to predict target miRNAs and chemicals of hub genes, and constructed the mRNA-miRNA co-expressed, protein-chemical interaction networks based on the interaction information using Cytoscape.

\section{Construction of ceRNA networks}


StarBase (version 3.0) (http://starbase.sysu.edu.cn/index.php) was used to predict IncRNAs and

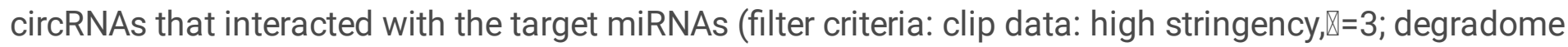
data: with or without degradome data). Finally, we used Cytoscape to constructed the ceRNA network based on the interactions among mRNAs, IncRNAs, miRNAs, and circRNAs.

\section{Tissue and intracellular distribution}

We used the Human Protein Atlas (HPA) Database (https://www.proteinatlas.org/) to find out the subcellular distribution of key genes and their expression in bone marrow, blood and muscle tissues (normalized expression, NX). The gene expression scores of key genes in bone marrow, blood, trabecular bone tissue, layer of synovial tissue, skeletal muscle tissue, and cartilage tissue were obtained from the Bgee database (https://bgee.org).

\section{Statistics analysis}

The R software (version3.6.2) was used to perform stastical analysis which was visualized by using ggplot2 package. The $T$ test was used to compare the difference of hub genes between two groups. The $\mathrm{R}$ software $\mathrm{PROC}$ package was used for ROC analysis and ggplot2 package was used to draw ROC curve.

\section{Ethics statement}

All analyses were based on the public GEO database, we did not need the informed consent of the patients, thus no ethical approval and patient consent are required.

\section{Results}

\section{Identifcation of DEGs}

The dataset GSE123568 including 30 ONFH samples and 10 normal control samples was used for the analysis and identification of DEGs. Comparing with the genes in the normal control samples, we identified a total of 568 DEGs in the ONFH samples, including 221 down-regulated and 347 up-regulated genes. Next, a heat map and volcano plot analysis were used to visualize these DEGs, as shown in Fig. 2a-b. The median of each sample was basically on one horizontal line, so the degree of normalization among the samples was great (Fig. 2c).

\section{Enrichment analysis}

Functional and pathway enrichment analysis was performed using the $\mathrm{R}$ software clusterProfiler Package. The screening criteria for significant gene sets were $p<0.05$ and False discovery rate (FDR) 
$<0.25$. We observed that most of the enriched gene sets were associated with the innate immune responses, thrombosis and signal transduction (Table 1). Next, GO, KEGG pathway and Reactome enrichment analyses were performed for DEGs using the $\mathrm{R}$ software clusterProfiler package. We will assess the DEGs enrichment pathways from multiple perspectives. Based on the $Q$ value $<0.05$, we selected the top two, three, one and four biological processes (BP), cellular components (CC), molecular functions (MF), and KEGG pathways and displayed them in chordal curve, circle diagram, bubble plots, and histogram (Fig. 3a-e). The GO enrichment analysis of DEGs also shows that ONFH samples have a stronger neutrophil response than control samples, including neutrophil activation, neutrophil activation involved in immune response, neutrophil mediated immunity, and neutrophil degranulation. KEGG pathway enrichment analysis showed that DEGs were enriched for osteoclast differentiation, leishmaniasis, the interaction of viral proteins with cytokines and cytokine receptors, and chemokine signaling pathways. Particularly, nine pathways related to immunity and thrombosis were visualized in Fig. $3 f$.

\section{PPI network construction, MCODE cluster modules and hub gene identifcation}

The interaction network between proteins encoded by DEGs was constructed by STRING, consisting of 174 nodes and 1,510 edges, and visualized by Cytoscape (Fig. 4a). In this network, we identified four modules using MCODE plugin based on filtering criteria, as shown in Fig. 4b-e. Cluster 1 has the highest cluster score ( 24 nodes and 133 edges), followed by cluster 2 ( 11 nodes and 53 edges), cluster 3 (18 nodes and 55 edges), and cluster 4 ( 11 nodes and 15 edges). Fifteen hub genes such as cytochrome b245 beta chain (CYBB) and CD86 molecule (CD86) were selected by intersecting the results of five algorithms of cytohubba, including MCC, DMNC, degree, MNC, and clustering coefficients as showed in Table 2 (16). The fifteen hub genes may play an imprtant role in the pathogenesis of ONFH. Finally, we analysed the correlation among the fifteen hub genes by using ggplot2 $\mathrm{R}$ package. We found that most genes were positively correlated with each others (Fig. 4f).

\section{Table 2}

15 hub genes identifed by cytoHubba in PPI network. 


\begin{tabular}{|c|c|c|c|c|}
\hline $\begin{array}{l}\text { Gene } \\
\text { symbol }\end{array}$ & Description & $\log 2 \mathrm{FC}$ & $Q$ value & Regulation \\
\hline CYBB & cytochrome b- 245 beta chain & 1.200 & 0.002 & Up \\
\hline CD86 & CD86 molecule & 1.226 & $\begin{array}{l}2.65 \mathrm{E}- \\
04\end{array}$ & Up \\
\hline TYROBP & $\begin{array}{l}\text { transmembrane immune signaling adaptor } \\
\text { TYROBP }\end{array}$ & 1.180 & $\begin{array}{l}5.42 \mathrm{E}- \\
05\end{array}$ & Up \\
\hline TLR4 & toll like receptor 4 & 1.149 & $\begin{array}{l}8.58 \mathrm{E}- \\
04\end{array}$ & Up \\
\hline LILRB2 & leukocyte immunoglobulin like receptor B2 & 1.200 & $\begin{array}{l}2.21 \mathrm{E}- \\
04\end{array}$ & Up \\
\hline TLR2 & toll like receptor 2 & 1.381 & $\begin{array}{l}9.83 \mathrm{E}- \\
05\end{array}$ & Up \\
\hline TLR8 & toll like receptor 8 & 1.329 & $\begin{array}{l}9.60 \mathrm{E}- \\
05\end{array}$ & Up \\
\hline CCR1 & C-C motif chemokine receptor 1 & 1.123 & 0.003 & Up \\
\hline TLR1 & toll like receptor 1 & 1.098 & $\begin{array}{l}2.57 \mathrm{E}- \\
04\end{array}$ & Up \\
\hline ITGAX & integrin subunit alpha $X$ & 1.143 & $\begin{array}{l}1.97 \mathrm{E}- \\
04\end{array}$ & Up \\
\hline NCF2 & neutrophil cytosolic factor 2 & 1.242 & $\begin{array}{l}1.22 \mathrm{E}- \\
04\end{array}$ & Up \\
\hline TREM1 & triggering receptor expressed on myeloid cells 1 & 1.465 & $\begin{array}{l}1.25 \mathrm{E}- \\
04\end{array}$ & Up \\
\hline IRF8 & interferon regulatory factor 8 & 1.072 & $\begin{array}{l}3.31 \mathrm{E}- \\
05\end{array}$ & Up \\
\hline NOD2 & $\begin{array}{l}\text { nucleotide binding oligomerization domain } \\
\text { containing } 2\end{array}$ & 1.039 & 0.006 & Up \\
\hline PLEK & pleckstrin & 1.008 & $\begin{array}{l}1.77 \mathrm{E}- \\
04\end{array}$ & Up \\
\hline
\end{tabular}

\section{Construction of the miRNA-mRNA and protein-chemical interaction networks}


We used the NetworkAnalyst database to predict the target miRNAs and chemicals of hub genes. We obtained 81 target miRNAs for 7 central genes (Fig. 5a) and 8 target chemicals for 2 central genes (Fig. $5 b)$.

\section{ROC curve of 15 hub genes in the peripheral secrum samples (GSE123568)}

The $\mathrm{PROC}$ and ggplot2 $\mathrm{R}$ package were used to analyse and draw the ROC curve of 15 hub genes in the peripheral secrum samples. Finally, the hub genes with hige diagnostic value (area under the curve, AUC > 0.8) were selected as key genes for further analysis, including CD86 (AUC=0.847), IRF8 (AUC=0.877), ITGAX (AUC=0.847), LILRB2 (AUC=0.880), NCF2 (AUC=0.887), PLEK (AUC=0.863), TLR1 (AUC=0.847), TLR2 (AUC=0.860), TLR4 (AUC=0.837), TREM1 (AUC=0.863), and TYROBP (AUC=0.873) (Fig. 6). Hence, we hypothesized they were the biomarkers for the diagnosis of ONFH based on our present samples.

\section{The expression of 15 hub genes in the hip cartilage samples (GSE74089)}

We analysed the expression of 15 hub genes in the hip cartilage samples, and identitied 4 key genes NOD2 ( $P$ value: 0.002 in GSE123568, 0.007 in GSE 74089), PLEK ( $P$ value: $<0.001$ in GSE123568, 0.001 in GSE 74089), TLR2 (P value: $<0.001$ in GSE123568, 0.038 in GSE 74089), TREM1 (P value: $<0.001$ in GSE123568, <0.001 in GSE 74089). As showed in Fig. 7a-c, the expression of four key genes were all increased in the peripheral secrum samples (GSE123568) and hip cartilage samples (GSE74089). Finally, three hub genes (PLEK, TREM1, TLR2) whose AUC (ROC) $₫ 0.8$ and expression trend was verified in GSE74089 was selected as key genes in the procession of ONFH.

\section{Tissue and intracellular distribution of PLEK, TREM1 and TLR2}

We compared the gene expression score of three key genes in Bgee database and the gene expression level of three key genes in HPA database. We found that, in gene expression level, PLEK was highly expressed in bone marrow and lowly in cartilage, TLR2 and TREM1 were highly expressed in blood and lowly in skeletal muscle tissue (Fig. 8a). In gene expression scores, PLEK was highly expressed in bone marrow and lowly in muscle tissues, TLR2 and TREM1 were highly expressed in blood and lowly in muscle tissue (Fig. 8b). Finally, we explored the intracellular distribution of key genes, the results showed that PLEK mainly expressed in nucleus, TREM1 in dictyosome, and TLR2 in nucleoplasm and mitochondria (Fig. 8c). 


\section{Prediction of target ncRNAs and construction of ceRNA networks}

It is well known that miRNAs induce gene silencing and down-regulate the gene expression by binding mRNAs. However, circRNA and IncRNA can up-regulate the expression of gene by binding miRNAs and competing with mRNAs. Such interactions between mRNAs, miRNAs, circRNAs, and IncRNAs are known as ceRNA networks (12). We selected ncRNAs present in most miRNA prediction results as our predicted circRNAs and IncRNAs. Finally, we obtained 13 target IncRNAs and 27 target circRNAs for PLEK 4 target miRNAs; and 13 target circRNAs and 19 target IncRNAs for TLR2 8 target miRNAs. But the predicted miRNAs of TREM1 was absent in NetworkAnalyst database. So only two ceRNA networks were constructed by Cytoscape based on the prediction results in the end (Fig. 9a-b). Subsequently, we conducted a literature search and selected two reproted down-regulated IncRNAs and one down-regulated miRNA in ONFH, one up-regulated miRNA in senescence of vascular smooth muscle cells, one downregulated miRNA in processe of osteoblast differentiation, and one circRNA that presented in most miRNA prediction results of TLR2. Finally, we proposed four potential RNA regulatory pathways of ONFH: MALAT1 (metastasis associated lung adenocarcinoma transcript 1)-miR-146b-5p-TLR2, MALAT1- miR664b-3p-PLEK, NORAD (non-coding RNA activated by DNA damage )-miR-106b-5p-TLR2, and MSM01(methylsterol monooxygenase 1)-miR-106b-5p-TLR2 (Fig. 9c).

\section{Discussion}

Although the etiology of traumatic ONFH has been specific, the pathogenesis underlying non-traumatic ONFH remains unclear. The diagnosis and treatment for early ONFH can effectively delay the process and significantly improve the life quality of ONFH patients. However, it is difficult to diagnose early ONFH owning to the lack of biomarkers, and effective drugs for ONFH are still lacking. This work explored the mechanisms of ONFH disease development at the transcriptome level and potential biomarkers for the early diagnosis and treatment of ONFH.

Immune disorder is closely related to the development of ONFH. For example, previous studies have found that IL-1 $a$ and TNF-a promote the development of ONFH, and IL-23 and IL-33 are vital for the diagnosis of ONFH (17-20). In our study, we screened out 568 DEGs by comparing gene expression of serum between ONFH patients and normal controls. GO enrichment analysis of DEGs revealed several immune-related pathways, such as nuetrophil degranulation, nuetrophil mediated immunity, nuetrophil activation involed in immune response, nuetrophil activation, signaling by interleukins, and class I MHC mediated antigen processing presentation. Furthermore, signaling pathways related to thrombosis and osteoclast processing are also involved, such as osteoclast differentiation and platelet activation signaling and aggregation. The GO enrichment results indicated these DEGs and pathways may be related to the development of ONFH. For example, Po'sa'n found that platelet activation was significantly higher in patients with ONFH compared with that in healthy controls (21). 
In our study, we found that the expression of NOD2, PLEK, TLR2 and TREM1 were up-regulated in ONFH patients (serum and hip cartilage), and the AUCs of PLEK (AUC=0.863), TLR2 (AUC=0.860) and TREM1 $(A U C=0.863)$ are more than 0.8. Therefore, we hypothesize that PLEK, TLR2 and TREM1 may be biomarkers for early diagnosis of ONFH and play a signifcant role in the pathogenesis of ONFH based on available samples. According to the Bgee and HPA online database, we found that the expression of PLEK, TLR2, and TREM1 in bone marrow and blood was higher than that in soft tissues such as muscle tissues. Furthermore, we constructed ceRNA networks and an mRNA-miRNA co-expression network to elucidate the pathogenesis of ONFH at the transcriptome level.

PLEK, is also called pleckstrin, is a protein kinase C (PKC) substrate induced by macrophages to express. It is known to play an important role in the secretion and activation of the pro-inflammatory cytokines IL$1 \beta$ and TNF- $\alpha(22,23)$. Studies have indicate that PLEK is significantly overexpressed in cardiovascular disease, and is a crucial protein for platelet aggregation, activation, and degranulation (24-26). According to BioGPS, PLEK is mainly expressed in nucleus. Although there are not studies that elucidated the function of PLEK in ONFH, our study found that PLEK was significantly up-regulated in serum and hip cartilage of ONFH patients, and has a high diagnostic value in $\mathrm{OFNH}(\mathrm{AUC}=0.863)$.

TLR2, is also known as toll like receptor 2, plays a fundamental role in pathogen recognition and activation of innate immunity and has been implicated in the pathogenesis of several autoimmune diseases according to the NCBI gene database. According to BioGPS, TLP2 is highly expressed in blood. At present, TLR2 has not been reported in ONFH-related studies. It is reported that the absence of TLR2 on bone marrow cells supports angiogenic processes and TLR2 promotes the atherosclerosis process (27, 28). In our study, TLR2 was up-regulated in serum and hip cartilage of ONFH patients compared with normal controls. It was predicted that miR-146a-5p and miR-106b-5p targeted at TLR2. MiR-146a-5p is down-regualated in ONFH and miR-106b-5p supresse the process of osteogenesis $(29,30)$. These results all refect that TLR2 may play an important role in the osteogenesis and the disease progression of ONFH.

TREM1, is also called triggering receptor expressed on myeloid cells 1, encodes a receptor belonging to the Ig superfamily that is expressed on myeloid cells. TREM1 also amplifies neutrophil and monocytemediated inflammatory responses (31). Similar to PLEK, TERM1 is also highly expressed in blood. And TREM1 is specifcally expressed in dictyosome composed of many flattened vesicles whose main function is secretion (32). A study by Yonatan Edel et al. reported that plasma TREM1 levels are significantly increased in patients with thrombotic primary antiphospholipid syndrome (33). It is known that intravascular thrombotic is possible pathogenesis of ONFH (34). So TREM1 may play an important role in the pathogenesis of ONFH. Besides, it also found that TREM1 promotes atherosclerosis and thrombin generation $(35,36)$. In our study, we identifed that TREM1 was significantly up-regulated in $\mathrm{ONFH}$ and has a high diagnostic value in ONFH (AUC=0.863). We considered TREM1 as a novel and effective biomarker for the diagnosis and treatment of ONFH.

Furthermore, the target IncRNAs and circRNAs of target miRNAs were predictd for PLEK and TLR2, and ceRNA networks were constructed with Cytoscape. The ceRNA networks revealed the potential 
pathogenesis of ONFH at the transcriptome level. Then we conducted a literature research to select potential miRNAs, IncRNAs, and circRNAs. Among the miRNAs, we selected down-regulated miRNA in ONFH: miRNA-146a-5p (in osteoblast), up-regulated miRNA in senescent vascular smooth muscle cells: miR-664b-3p, and miRNA suppressing osteogenesis: miR-106b-5p (29, 30, 37). Among the IncRNAs, we selected down-regulated IncRNAs in ONFH: MALAT1 (in serum), NORAD (in bone marrow mesenchyml stem cell) $(38,39)$. Therefore, we proposed three potential RNA regulatory pathways in the process of ONFH: MALAT1-miR-146b-5p-TLR2/ MALAT1-miR-664b-3p-PLEK/ NORAD-miR-106b-5p-TLR2. Finally, we selected one circRNA that presented in most miRNA prediction results of TLR2: MSM01, and proposed a potential RNA regulatory pathway: MSM01-miR-106b-5p-TLR2.

As far as I know, this is a novel study to explore effective biomarkers in the diagnosis and treatment of ONFH. Our study provides insights into the pathogenesis of ONFH. Of course, the sample size used for analysis in our study was relatively small and the prediction of ncRNA was just based on online database. Therefore, we have planed further study to increase sample size and conduct experiment to verify our views.

\section{Conclusion}

Our work identifed three crucial genes, PLEK, TLR2, and TREM1, as potential biomarkers for the diagnosis and treatment of ONFH and provided insight into the pathogenesis of ONFH at the transcriptome level. In addition, we propose that MALAT1-miR-146b-5p-TLR2, MALAT1- miR-664b-3p-PLEK, NORAD-miR-106b5p-TLR2, and MSM01-miR-106b-5p-TLR2 are potential RNA regulatory pathways in the disease progression of ONFH.

\section{Abbreviations}

DEGs: differentially expressed genes; PPI: protein-protein interaction; ONFH: osteonecrosis of the femoral head; SONFH: steroid-induced ONFH; ceRNA: competitive endogenous RNA; GEO: Gene Expression Omnibus; GO: Gene Ontology; KEGG: Kyoto Encyclopedia of Genes and Genomes; HPA: Human Protein Atlas; ROC: receiver operating characteristic; GSEA: Gene Set Enrichment Analysis; NES: normalized enrichment score; FDR: false discovery rate; MCODE: Minimal Common Oncology Data Elements; MNC: Maximum Neighborhood Component; MCC: Maximal Clique Centrality; IncRNA: long noncoding RNA; circRNA: circular RNA; nx: normalized expression; BP: biological processes; CC: cellular components; MF: molecular functions; AUC: area under the curve; PKC: protein kinase C; MALAT1: metastasis associated lung adenocarcinoma transcript 1; NORAD: non-coding RNA activated by DNA damage; MSM01: methylsterol monooxygenase 1; CYBB: cytochrome b-245 beta chain; CD86: CD86 molecule

\section{Declarations}

\section{Acknowledgements}




\section{Authors' contributions}

Conception and design: D Yang, Z Zhang; Administrative support: Z Zhang; Provision of study materials: D Yang, Z Zhang; Collection and assembly of data: D Yang, S Chen, $Y$ Chen; Data analysis and interpretation: D Yang, S Chen, Y Chen, B Wang, G Zhang; Manuscript writing: All authors; Final approval of manuscript: All authors.

\section{Funding}

None.

\section{Availability of data and materials}

We state that the data will not be shared since all the raw data are present in the article.

\section{Ethics approval and consent to participate}

Not applicable.

\section{Consent for publication}

Not applicable.

\section{Competing interests}

The authors declare that they have no competing interests.

\section{Author details}

${ }^{1}$ Department of Orthopedics, the Second Hospital of Shanxi Medical University, No.382 Wuyi Road Taiyuan, China, 030000; ${ }^{2}$ School of Public Health, Xi'an Jiaotong University, No.76 Yanta xi Road, Xi'an, China, 710000 .

\section{References}


1. Tian L, Sun S, Li W, et al. Down-regulated microRNA-141 facilitates osteoblast activity and inhibits osteoclast activity to ameliorate osteonecrosis of the femoral head via up-regulating TGF-beta2. Cell Cycle 2020;19(7):772-86.

2. Mont MA, Hungerford DS. Non-traumatic avascular necrosis of the femoral head. J Bone Joint Surg Am 1995;77(3):459-74.

3. Maruyama M, Nabeshima A, Pan CC, et al. The effects of a functionally-graded scaffold and bone marrow-derived mononuclear cells on steroid-induced femoral head osteonecrosis. Biomaterials 2018;187:39-46.

4. Hao Y, Guo H, Xu Z, et al. Meta-analysis of the potential role of extracorporeal shockwave therapy in osteonecrosis of the femoral head. J Orthop Surg Res 2018;13(1):166.

5. Durbin RP. Letter: Acid secretion by gastric mucous membrane. Am J Physiol 1975;229(6):1726.

6. Tan G, Kang PD, Pei FX. Glucocorticoids affect the metabolism of bone marrow stromal cells and lead to osteonecrosis of the femoral head: a review. Chin Med J (Engl) 2012;125(1):134-9.

7. Wang A, Ren M, Wang J. The pathogenesis of steroid-induced osteonecrosis of the femoral head: A systematic review of the literature. Gene 2018;671:103-9.

8. Li G, Liu H, Zhang X, et al. The protective effects of microRNA-26a in steroid-induced osteonecrosis of the femoral head by repressing EZH2. Cell Cycle 2020;19(5):551-66.

9. Demircioglu D, Cukuroglu E, Kindermans M, et al. A Pan-cancer Transcriptome Analysis Reveals Pervasive Regulation through Alternative Promoters. Cell 2019;178(6):1465-77 e17.

10. Kaczkowski B, Tanaka Y, Kawaji H, et al. Transcriptome Analysis of Recurrently Deregulated Genes across Multiple Cancers Identifies New Pan-Cancer Biomarkers. Cancer Res 2016;76(2):216-26.

11. Han N, Li Z. Non-coding RNA Identification in Osteonecrosis of the Femoral Head Using Competitive Endogenous RNA Network Analysis. Orthop Surg 2021;13(3):1067-76.

12. Salmena L, Poliseno L, Tay Y, et al. A ceRNA hypothesis: the Rosetta Stone of a hidden RNA language? Cell 2011;146(3):353-8.

13. Davis S, Meltzer PS. GEOquery: a bridge between the Gene Expression Omnibus (GEO) and BioConductor. Bioinformatics 2007;23(14):1846-7.

14. Yu G, Wang LG, Han Y, et al. clusterProfiler: an R package for comparing biological themes among gene clusters. OMICS 2012;16(5):284-7.

15. Walter W, Sanchez-Cabo F, Ricote M. GOplot: an R package for visually combining expression data with functional analysis. Bioinformatics 2015;31(17):2912-4.

16. Chin $\mathrm{CH}$, Chen $\mathrm{SH}, \mathrm{Wu} \mathrm{HH}$, et al. cytoHubba: identifying hub objects and sub-networks from complex interactome. BMC Syst Biol 2014;8 Suppl 4:S11.

17. El-Tahan RR, Ghoneim AM, El-Mashad N. TNF-alpha gene polymorphisms and expression. Springerplus 2016;5(1):1508.

18. Oppmann B, Lesley R, Blom B, et al. Novel p19 protein engages IL-12p40 to form a cytokine, IL-23, with biological activities similar as well as distinct from IL-12. Immunity 2000;13(5):715-25. 
19. Parham C, Chirica M, Timans J, et al. A receptor for the heterodimeric cytokine IL-23 is composed of IL-12Rbeta1 and a novel cytokine receptor subunit, IL-23R. J Immunol 2002;168(11):5699-708.

20. Samara S, Kollia P, Dailiana Z, et al. Predictive role of cytokine gene polymorphisms for the development of femoral head osteonecrosis. Dis Markers 2012;33(4):215-21.

21. Posan E, Harsfalvi J, Szepesi K, et al. Increased platelet activation and decreased fibrinolysis in the pathogenesis of aseptic necrosis of the femoral head. Platelets 1998;9(3-4):233-5.

22. Ding Y, Kantarci A, Badwey JA, et al. Phosphorylation of pleckstrin increases proinflammatory cytokine secretion by mononuclear phagocytes in diabetes mellitus. J Immunol 2007;179(1):647-54.

23. Hasturk H, Kantarci A, Van Dyke TE. Oral inflammatory diseases and systemic inflammation: role of the macrophage. Front Immunol 2012;3:118.

24. Lundmark A, Davanian $\mathrm{H}$, Bage $\mathrm{T}$, et al. Transcriptome analysis reveals mucin 4 to be highly associated with periodontitis and identifies pleckstrin as a link to systemic diseases. Sci Rep 2015;5:18475.

25. Abrams CS, Zhao W, Belmonte E, et al. Protein kinase $\mathrm{C}$ regulates pleckstrin by phosphorylation of sites adjacent to the N-terminal pleckstrin homology domain. J Biol Chem 1995;270(40):23317-21.

26. Frobel J, Cadeddu RP, Hartwig S, et al. Platelet proteome analysis reveals integrin-dependent aggregation defects in patients with myelodysplastic syndromes. Mol Cell Proteomics 2013;12(5):1272-80.

27. Wagner NM, Bierhansl L, Butschkau A, et al. TLR2-deficiency of cKit+ bone marrow cells is associated with augmented potency to stimulate angiogenic processes. Int $\mathrm{J}$ Clin Exp Pathol 2013;6(12):2813-23.

28. Dunzendorfer S, Lee HK, Tobias PS. Flow-dependent regulation of endothelial Toll-like receptor 2 expression through inhibition of SP1 activity. Circ Res 2004;95(7):684-91.

29. Li P, Sun N, Zeng J, et al. Differential expression of miR-672-5p and miR-146a-5p in osteoblasts in rats after steroid intervention. Gene 2016;591(1):69-73.

30. Fang T, Wu Q, Zhou L, et al. miR-106b-5p and miR-17-5p suppress osteogenic differentiation by targeting Smad5 and inhibit bone formation. Exp Cell Res 2016;347(1):74-82.

31. Gaudet P, Livstone MS, Lewis SE, et al. Phylogenetic-based propagation of functional annotations within the Gene Ontology consortium. Brief Bioinform 2011;12(5):449-62.

32. Liu J, Huang Y, Li T, et al. The role of the Golgi apparatus in disease (Review). Int J Mol Med 2021;47(4).

33. Edel Y, Kliminski V, Pokroy-Shapira E, et al. Elevated plasma level of soluble triggering receptor expressed on myeloid cells- 1 is associated with inflammation activity and is a potential biomarker of thrombosis in primary antiphospholipid syndrome. Arthritis Res Ther 2019;21(1):10.

34. Xu J, Gong H, Lu S, et al. Animal models of steroid-induced osteonecrosis of the femoral head-a comprehensive research review up to 2018. Int Orthop 2018;42(7):1729-37. 
35. Di Pillo E, Carrasco K, Brustolin B, et al. Inhibition of triggering receptor expressed on myeloid cells-1 impairs thrombin generation. J Thromb Haemost 2020;18(2):454-62.

36. Joffre J, Potteaux S, Zeboudj L, et al. Genetic and Pharmacological Inhibition of TREM-1 Limits the Development of Experimental Atherosclerosis. J Am Coll Cardiol 2016;68(25):2776-93.

37. Nguyen DDN, Zain SM, Kamarulzaman MH, et al. Intracellular and exosomal microRNAome profiling of human vascular smooth muscle cells during replicative senescence. Am J Physiol Heart Circ Physiol 2021;321(4):H770-H83.

38. Jin Y, Zhu HX, Wei BF. Reduced serum and local LncRNA MALAT1 expressions are linked with disease severity in patients with non-traumatic osteonecrosis of the femoral head. Technol Health Care 2021;29(3):479-88.

39. Fu D, Yang S, Lu J, et al. LncRNA NORAD promotes bone marrow stem cell differentiation and proliferation by targeting miR-26a-5p in steroid-induced osteonecrosis of the femoral head. Stem Cell Res Ther 2021;12(1):18.

\section{Table}

Due to technical limitations, table 1 is only available as a download in the Supplemental Files section.

\section{Figures}

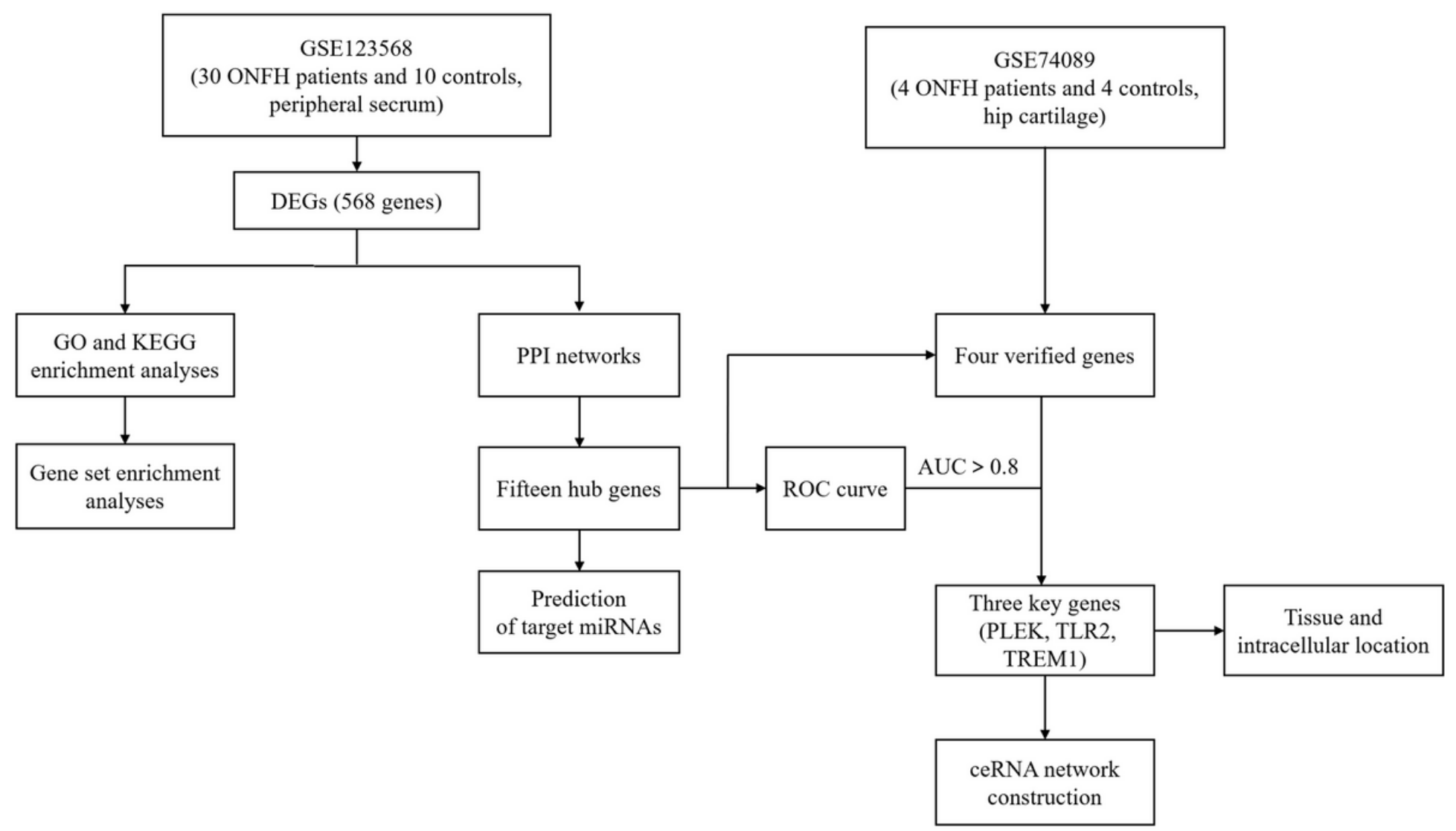

Figure 1 
The flow chart of the whole study.

A

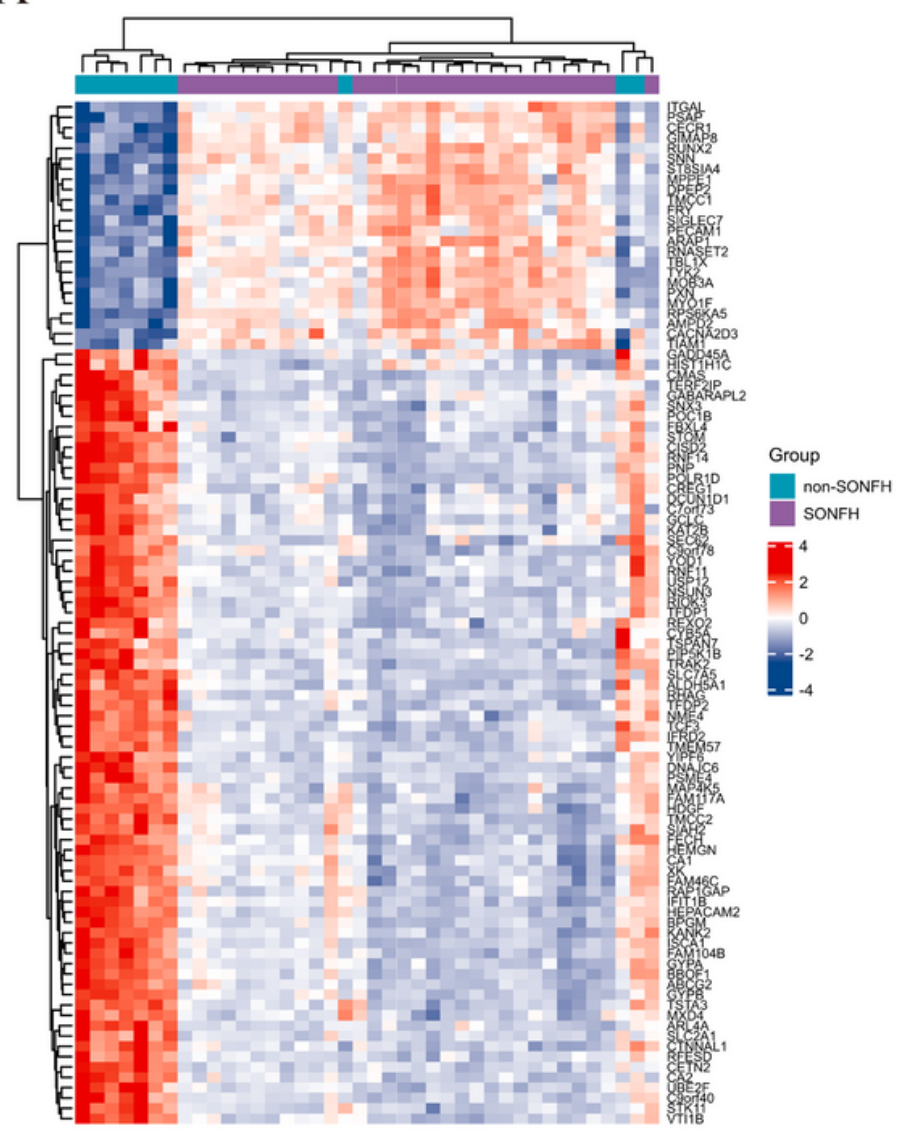

B

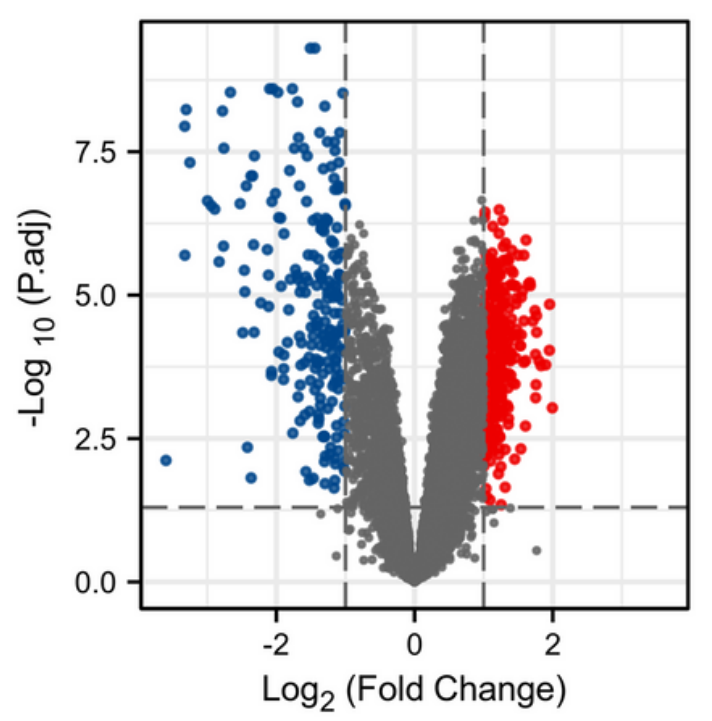

$\mathrm{C}$

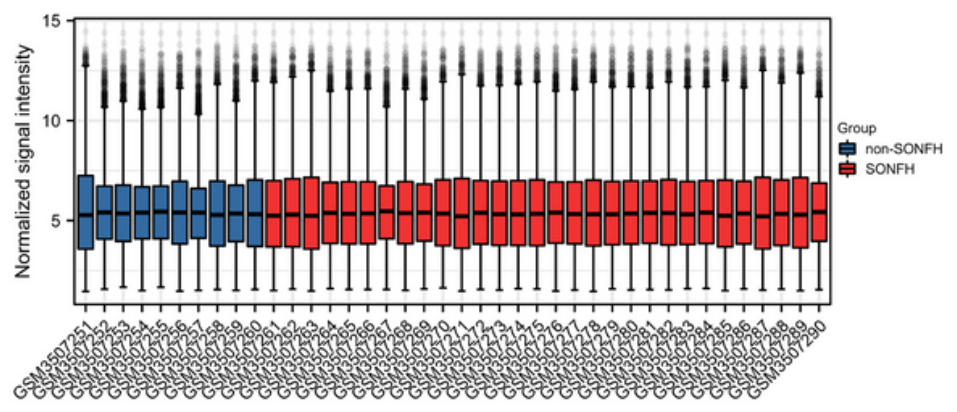

Figure 2

Identification of DEGs. (a) Heatmap plot of DEGs between the ONFH samples and normal control samples. (b) Volcano plot of DEGs between the ONFH samples and normal control samples. The red represents up-regulated genes and blue represents down-regulated genes. (c) The box diagram of ONFH samples and normal control samples for inter-sample normalization. The red represents the ONFH samples and blue represents normal control samples. DEGs, differentially expressed genes. 


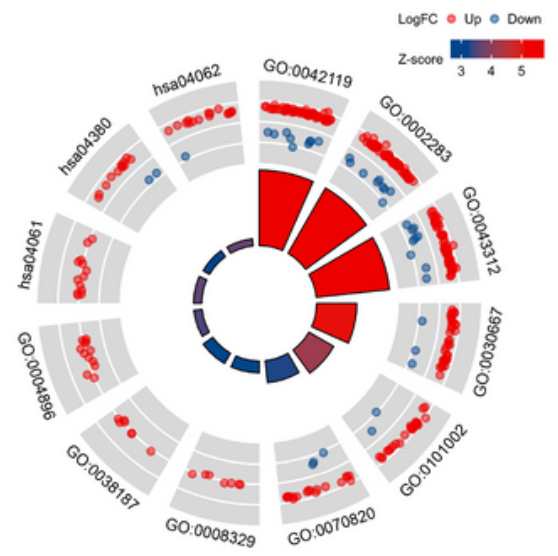

$\mathrm{C}$
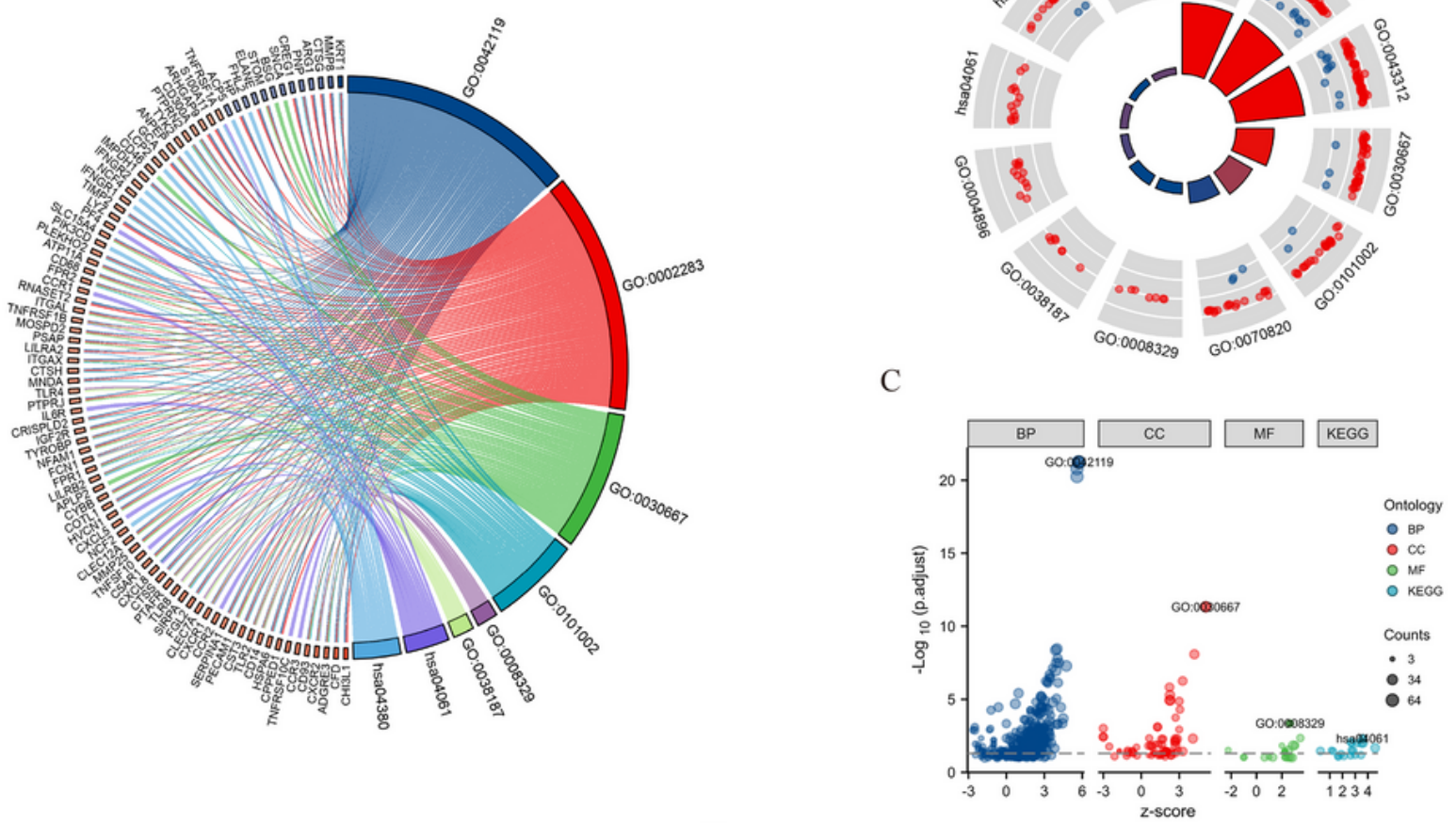

$\mathrm{D}$

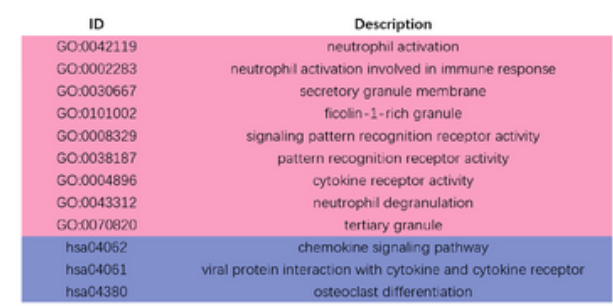

$\mathrm{F}$

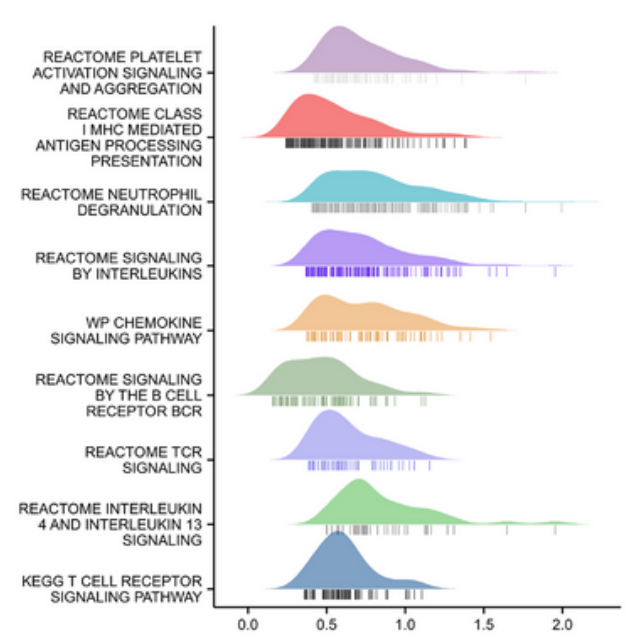

E

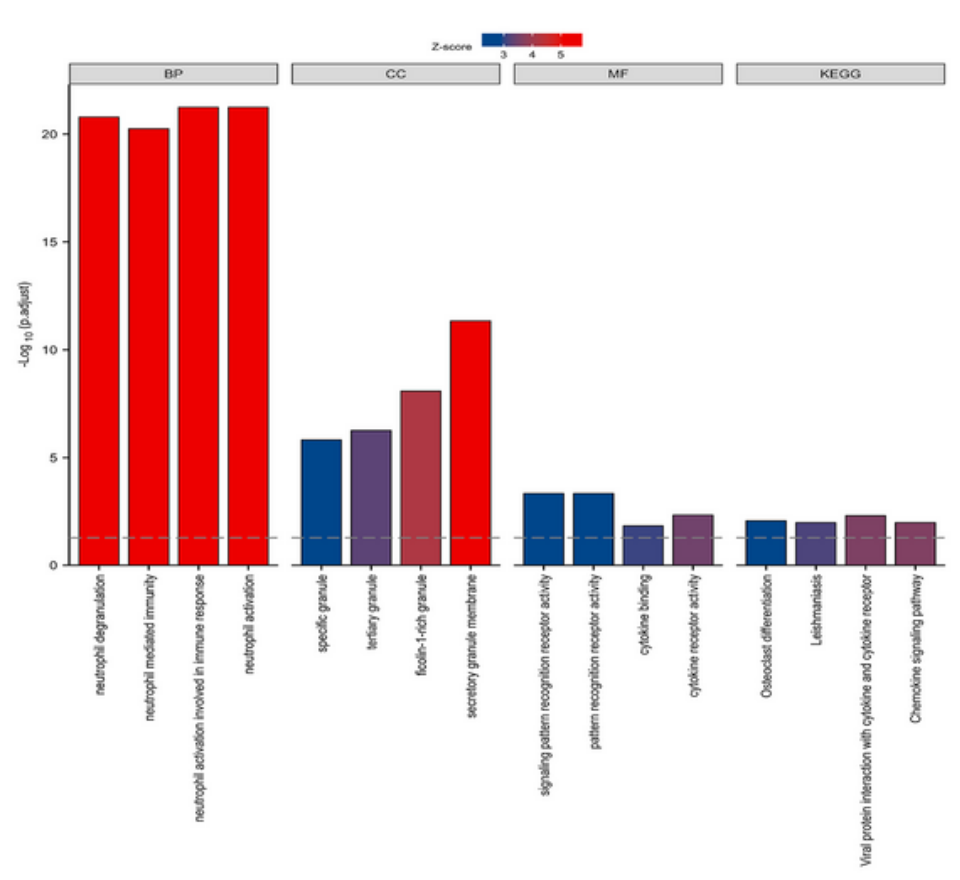

\section{Figure 3}

KEGG, GO pathway, and Reactome enrichment analyses of DEGs. (a) The chord plot showing the top two BP, CC, MF, and KEGG pathways. (b) The circle diagram showing the top three BP, CC, MF, and KEGG pathways. (c) The circle diagram showing the top three BP, CC, MF, and KEGG pathways. (d) The annotation of KEGG and $G O$ pathways. (e) The bubble plots showing the top one BP, CC, MF, and KEGG pathways. (f) The mountain plot showing nine pathways related to immunity and thrombosis. BP, 
biological processes; CC, cellular components; MF, molecular functions; GO, Gene Ontology; KEGG, Kyoto Encyclopedia of Genes and Genomes; DEGs, differentially expressed genes.

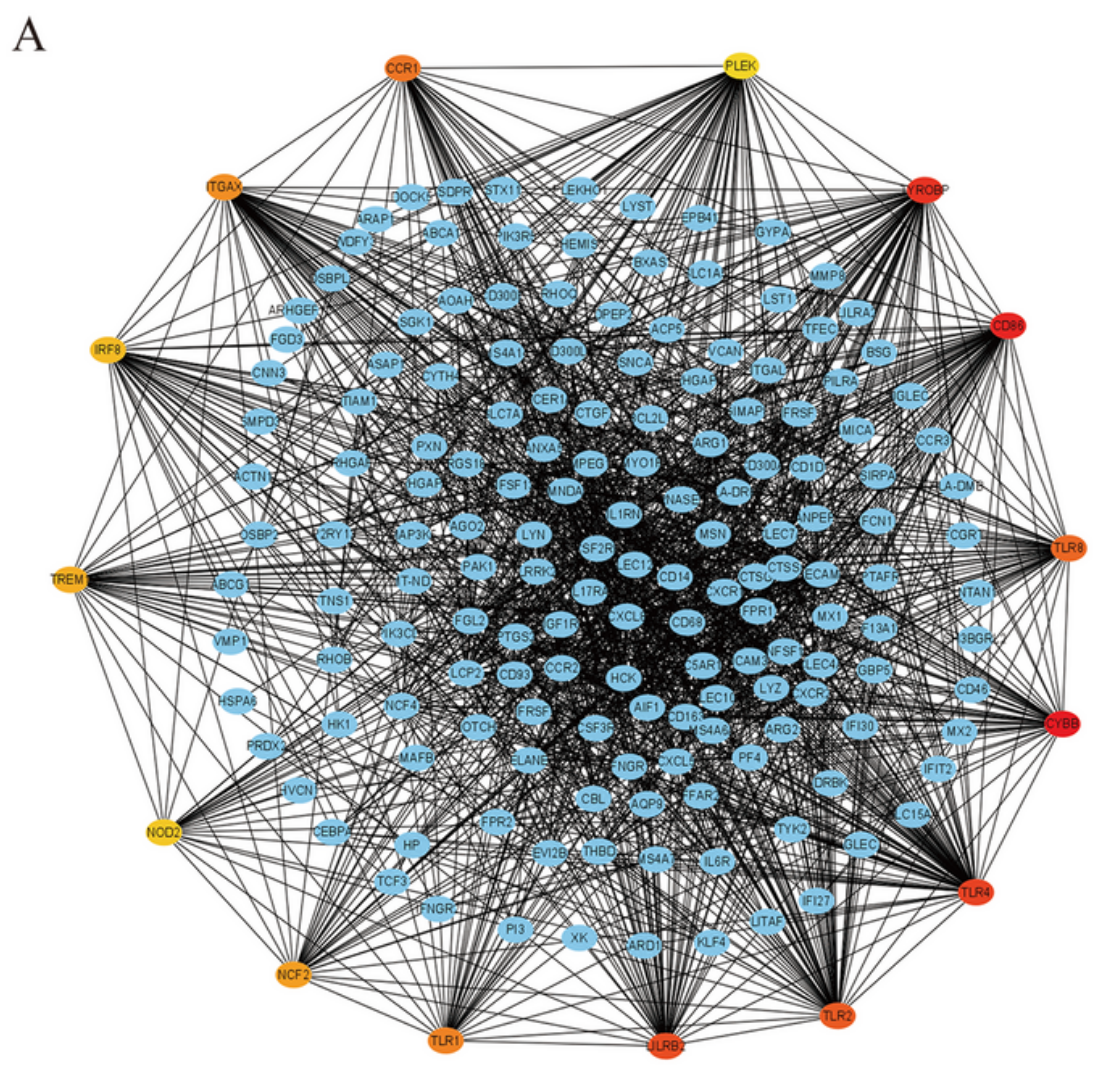

B

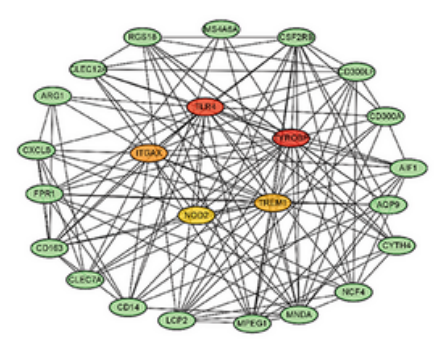

$\mathrm{D}$

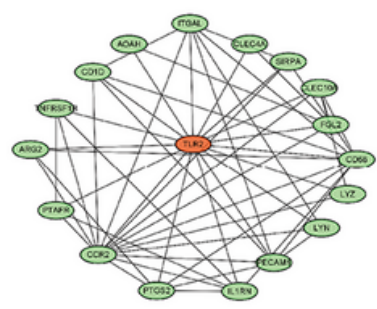

$\mathrm{C}$

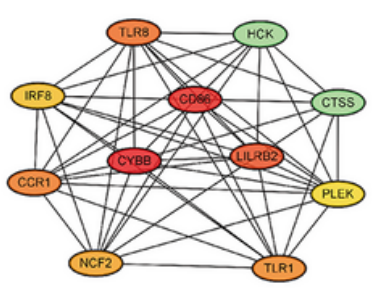

$\mathrm{E}$

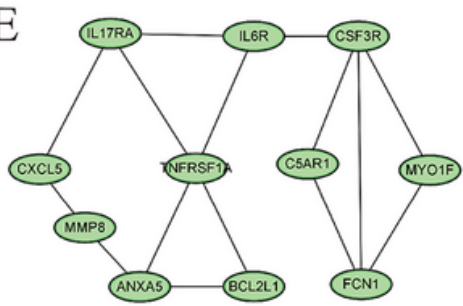

$\mathrm{F}$

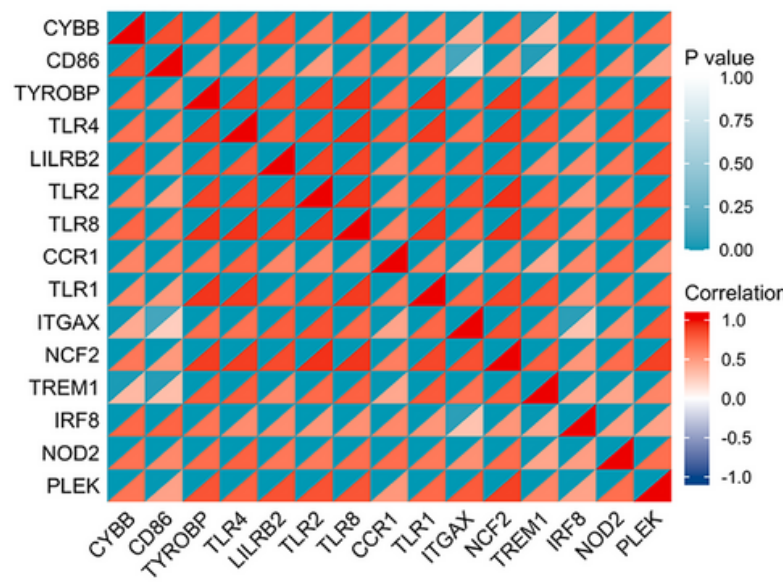

Figure 4

PPI network of DEGs constructed by cytoscape, four cluster modules extracted by MCODE, and correlation between hub genes with each other. Red, orange and yellow ellipses represent hub genes. (a) PPI network of DEGs (174 nodes and 1,510 edges). (B) Cluster1 (24 nodes and 133 edges) (b) Cluster2 (11 nodes and 53 edges) (c) Cluster 3 (18 nodes and 55 edges) (d) Cluster 4 (11 nodes and 15 edges). (e) 
The correlation between hub genes with each other. PPI, protein- protein interaction; DEGs, differentially expressed genes; MCODE, Minimal Common Oncology Data Elements.

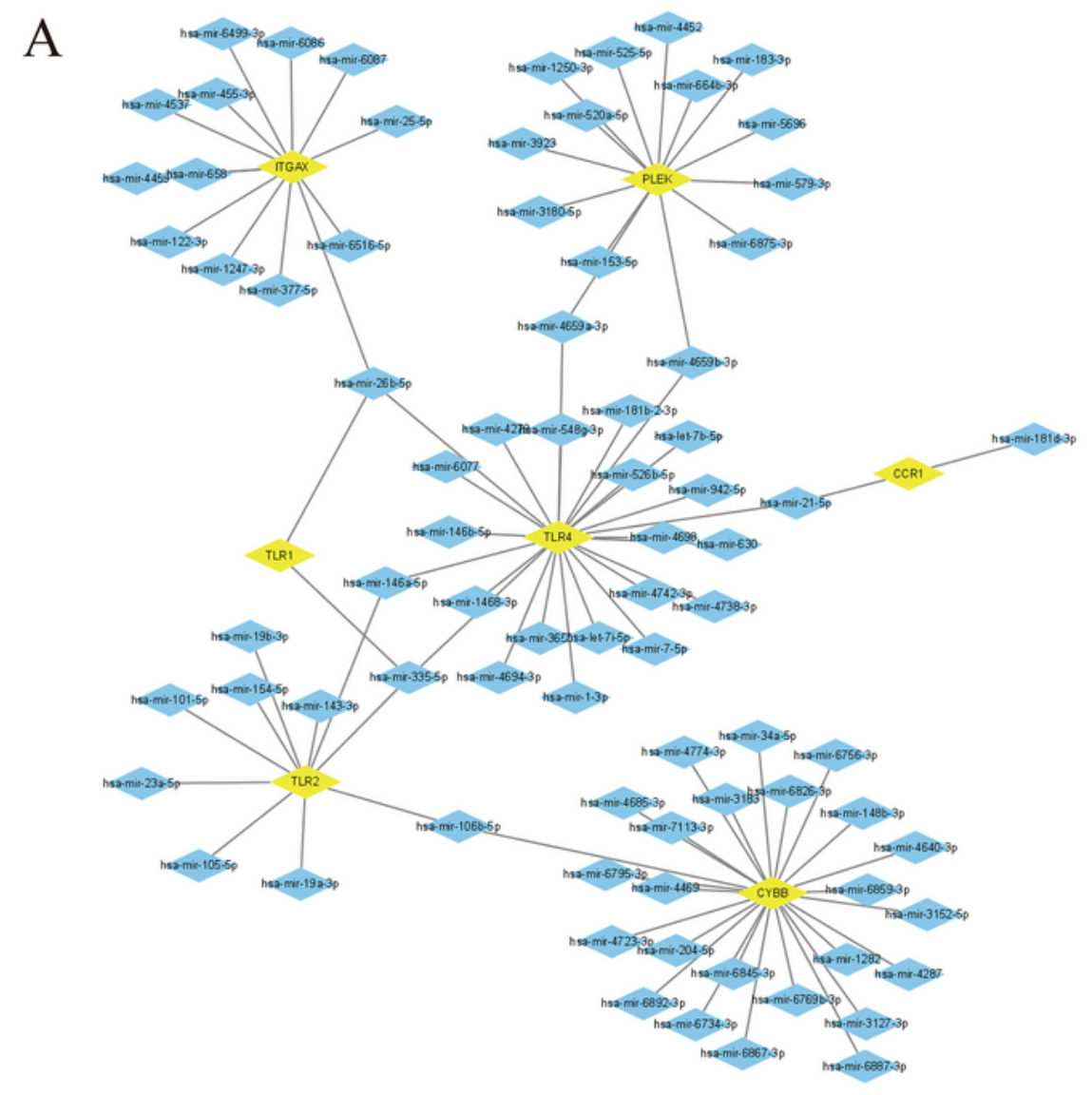

B

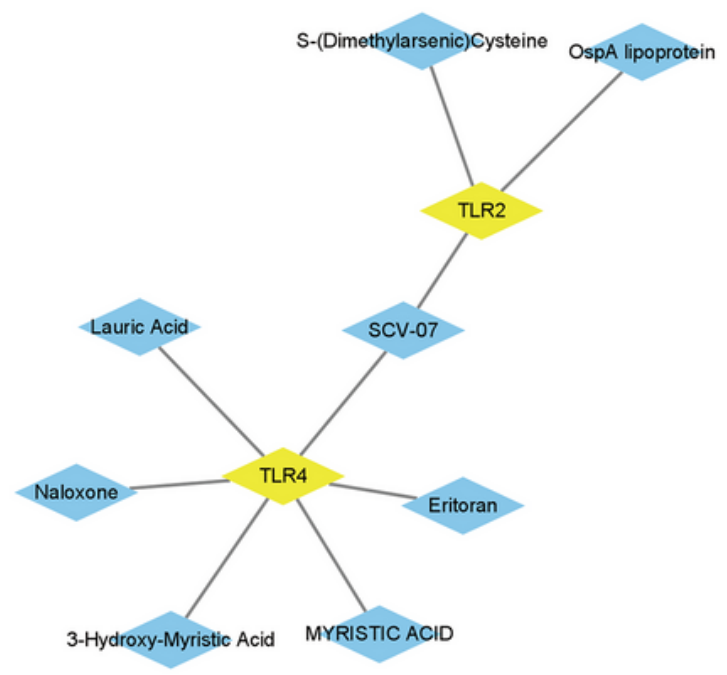

Figure 5

The mRNA-miRNA co-expressed and protein-chemical interaction networks constructed by Cytoscape. (a) The mRNA-miRNA co-expressed network including 81 target miRNAs for 7 hub genes. (b) The proteinchemical interaction network including 8 target chemicals for 2 hub genes. 

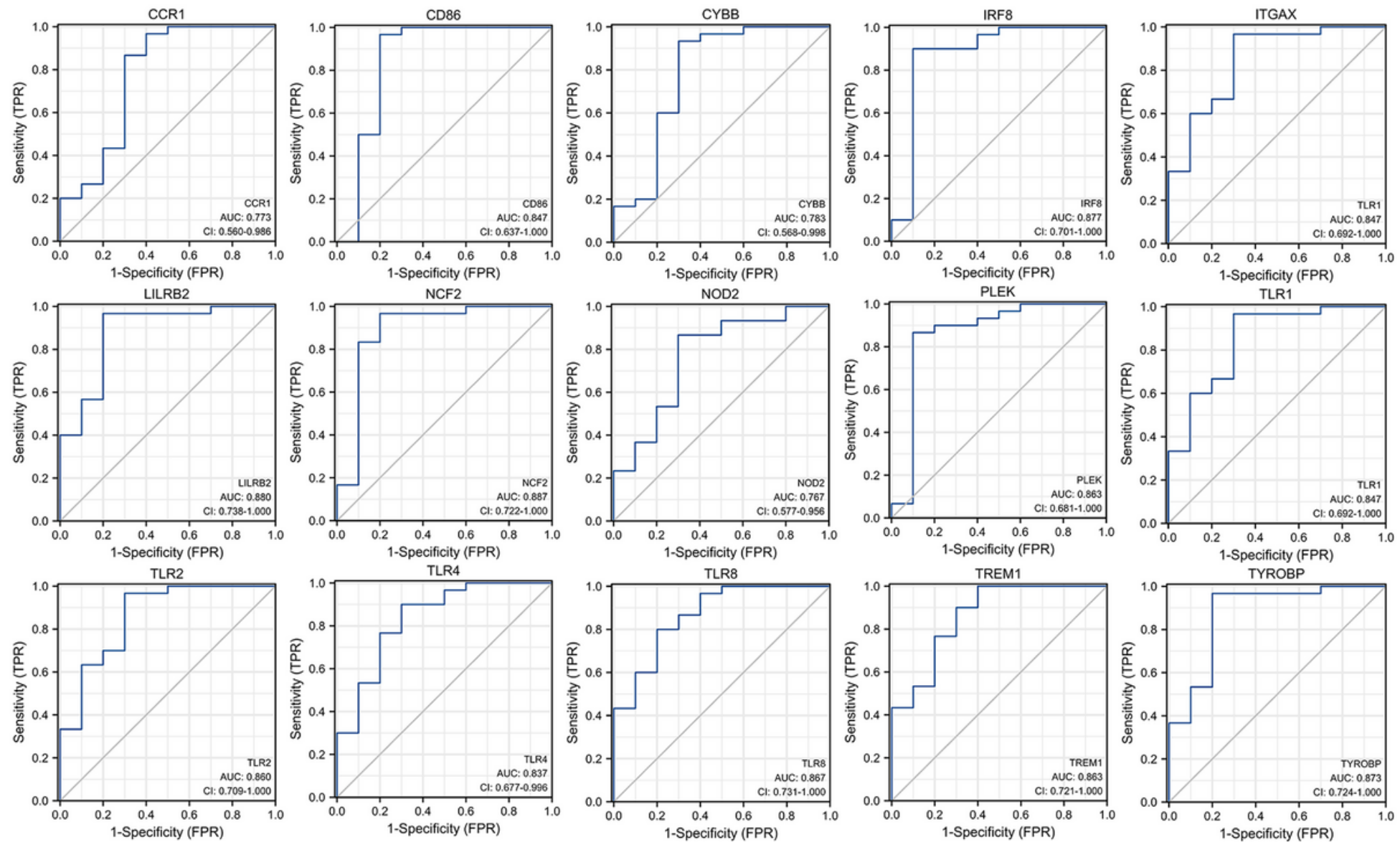

Figure 6

ROC curve of 15 hub genes in ONFH samples (GSE123568). The hub genes whose AUC was more than 0.8 were selected for further analysis. ROC, receiver operating characteristic; AUC, area under the curve. 
A
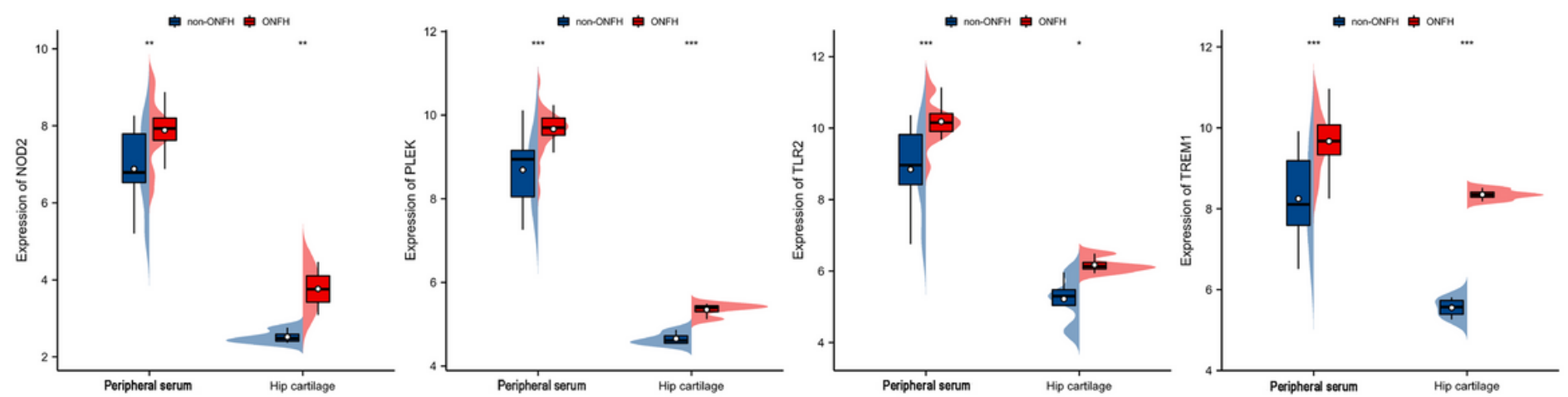

B
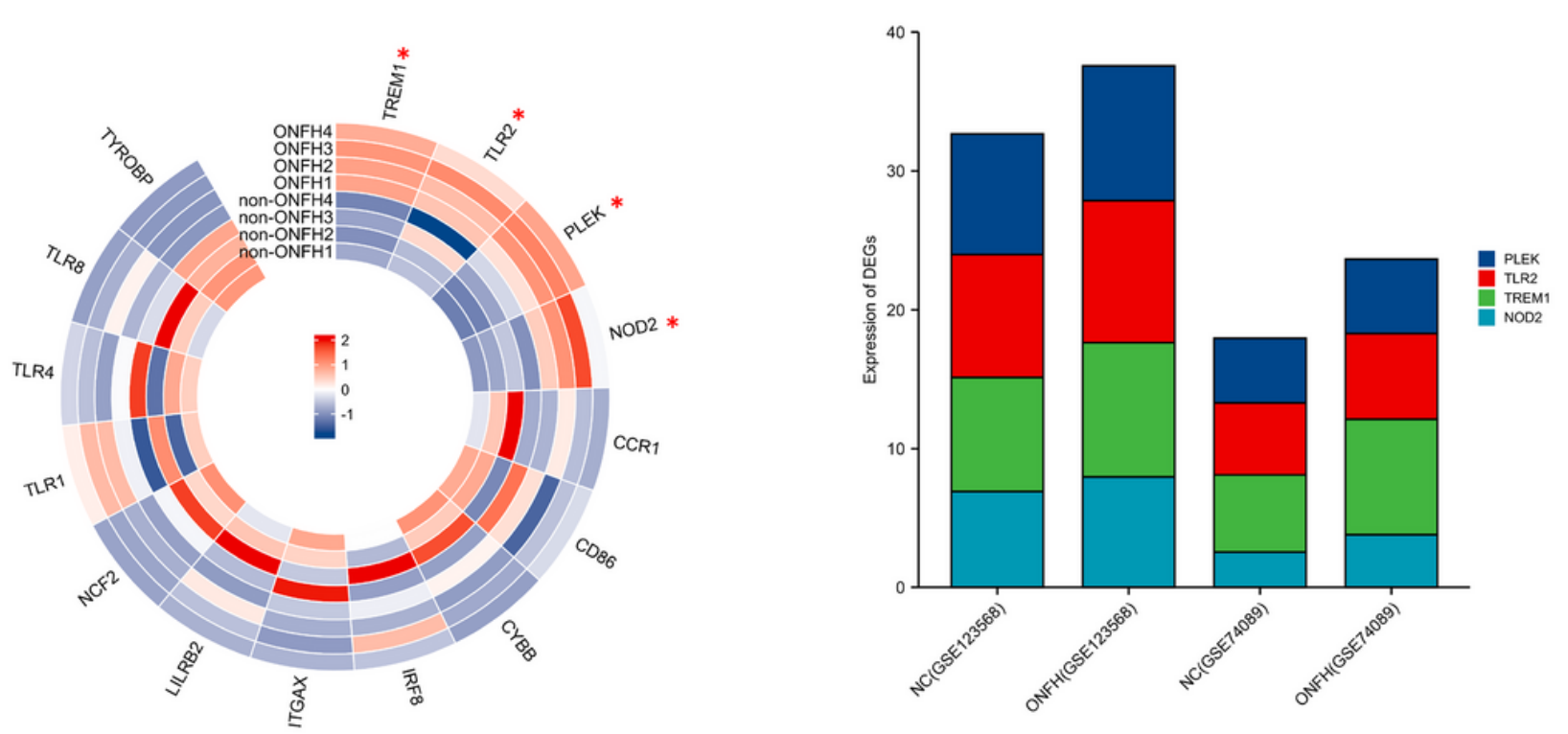

Figure 7

The expression of hub genes in peripheral serum (GSE123568) and hip cartilage (GSE74089). (a) The expression of NOD2, PLEK, TLR2, and TREM1 was up-regulated in peripheral serum and hip cartilage. (b) The expression of 15 hub genes in hip cartilage visualized as circular heat map. (c) The expression of NOD2, PLEK, TLR2, and TREM1 in GSE1 23568 and GSE74089 datasets visualized as histogram. NC,

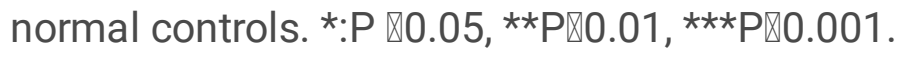


A

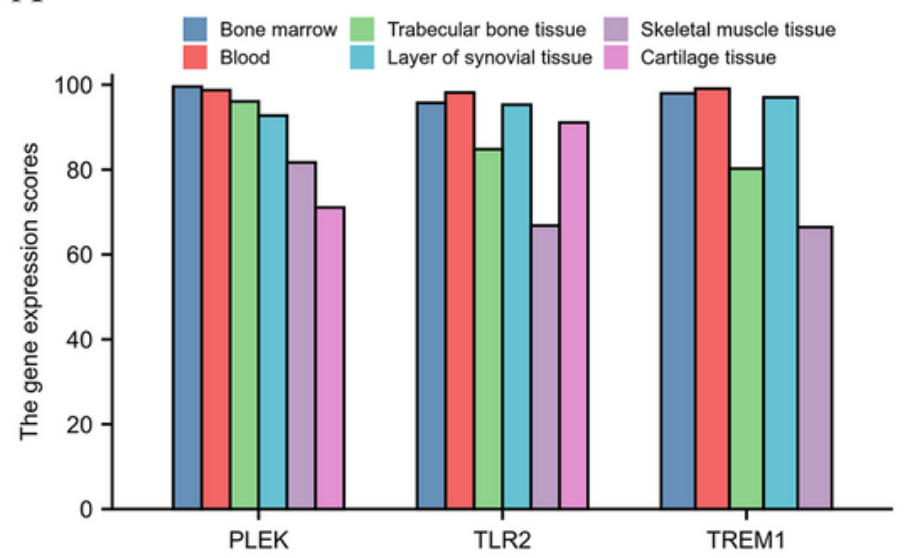

C

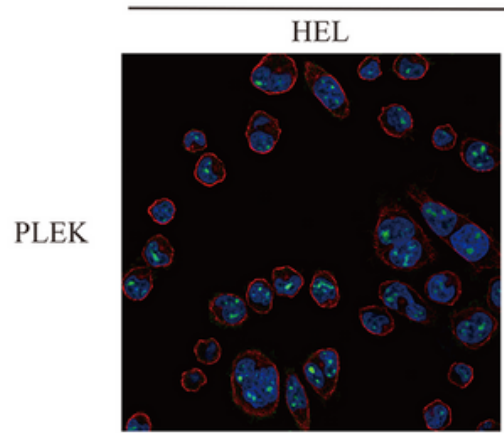

A-431

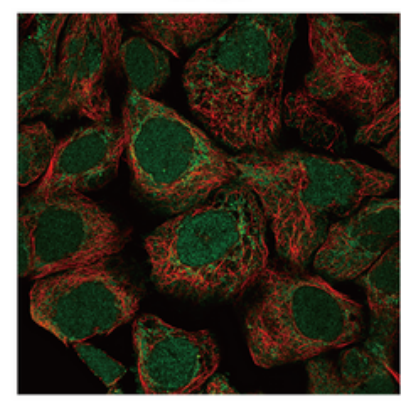

BJ

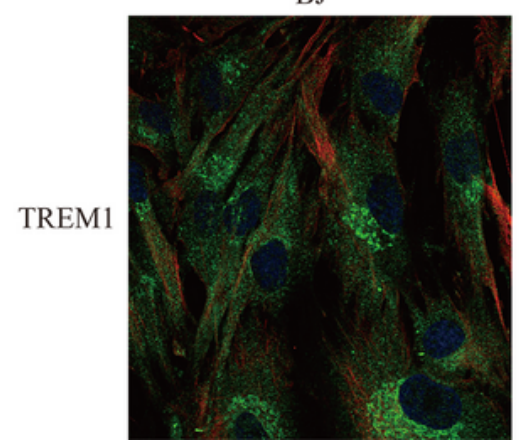

Cell line

RT4

SuSa
$\mathrm{B}$
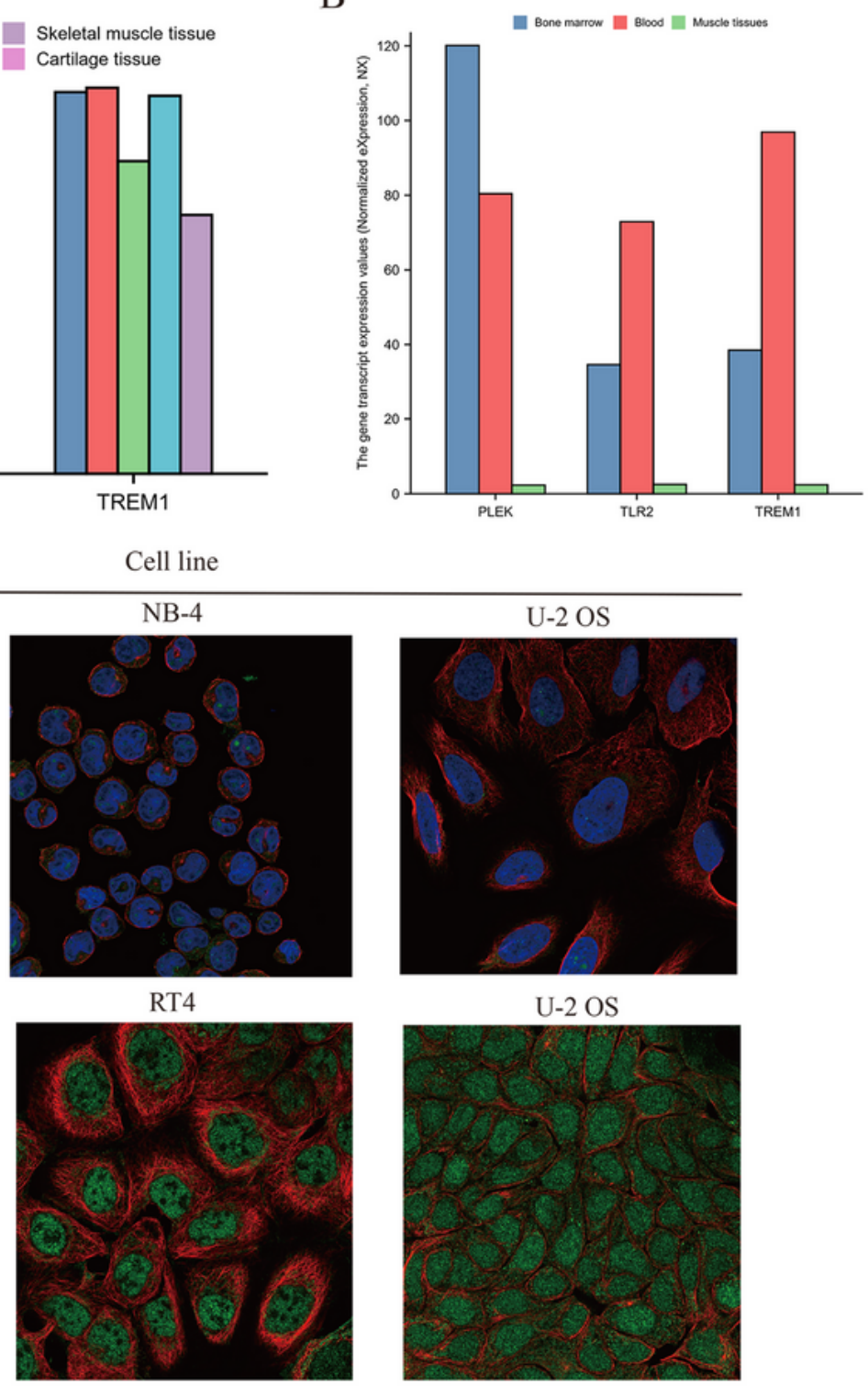

U-2 OS

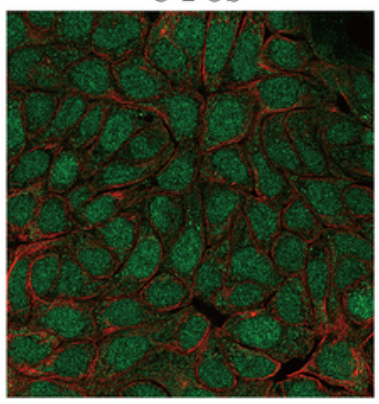

U-2 OS
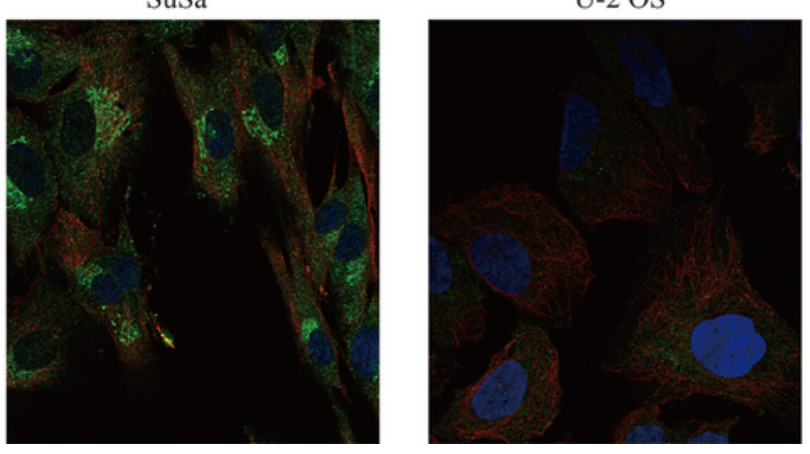

\section{Figure 8}

The tissue and intracellular distribution of PLEK, TREM1 and TLR2. (a) The gene expression scores of PLEK, TREM1 and TLR2 in bone marrow, trabecular bone tissue, skeletal muscle tissue, blood, layer of synovial tissue, and cartilage in in Bgee database. (b) The gene expression level of PLEK, TREM1 and TLR2 in HPA database. (c) The intracellular distribution of PLEK, TREM1 and TLR2. Green color represents the expression location of PLEK, TREM1 and TLR2. 

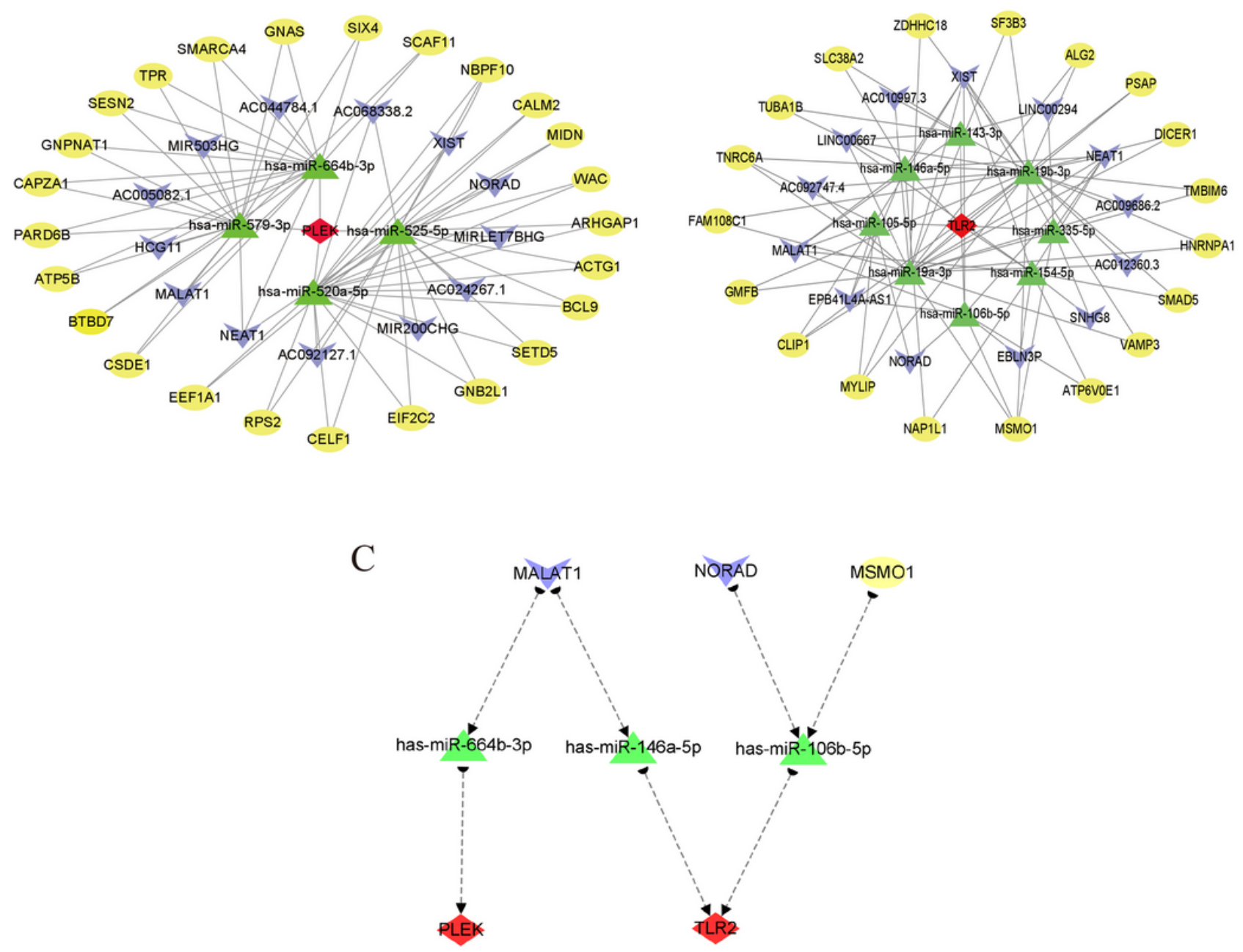

\section{Figure 9}

Two ceRNA networks of PLEK, and TLR2 and the potential RNA regulatory pathways. (a) ceRNA network of PLEK. (b) ceRNA network of TLR2. (c) MALAT1-miR-146b-5p-TLR2, MALAT1- miR-664b-3p-PLEK, NORAD-miR-106b-5p-TLR2, and MSM01-miR-106b-5p-TLR2. MALAT1, metastasis associated lung adenocarcinoma transcript 1; NORAD, non-coding RNA activated by DNA damage; MSMO1, methylsterol monooxygenase 1.

\section{Supplementary Files}

This is a list of supplementary files associated with this preprint. Click to download.

- Table1.docx 